\title{
Mathematical Model on Attack of Worm and Virus in Computer Network
}

\author{
Binay Kumar Mishra \\ Department of Physics, Veer Kunwar Singh University, Ara, India \\ drmishrabinay@gmail.com
}

\begin{abstract}
To curb the impact and transmission of worms and virus for simple mass action incidence rate in computer networks, $S I_{1} I_{2} R S$ (Susceptible, Infectious due to worm, Infectious due to virus, Recovered and Susceptible) model have been formed. Basic reproduction number, endemic equilibrium state and their stability are discussed for the simple mass action incidence. For locally and globally asymptotically stability of the infection free equilibrium state, Liapunov function is used. Numerical methods have been used to solve and simulate the system which will help us to understand the attacking behavior and transmission dynamics of malicious object in computer network, and efficiency of antivirus software.
\end{abstract}

Keywords: Epidemic model, Malicious Objects, Computer Network, Threshold, Global Stability

\section{Introduction}

Nothing is permanent in nature, everything that exist changes in course of time, and this very change gave us a fantastic world of cyber. It has brought sea-change in the society that we live in. Over the years, the internet technology has been continuously offering multiple functionalities and facilities. The augmentation of cyber technology has helped in data and information exchange to take at blazing speed which transforms the world into global village. It has made the life easier and world accessible with the touch of button. But everything is not well in cyber world; it is facing several challenges in the form of malicious objects. These malicious objects are worms, virus and Trojan horse. Malicious objects have tremendous influence on computer network. Currently, e-mail has become one of the main factors for the transmission of malicious objects. Transmission of malicious objects in computer network is epidemic in nature and is analogous to biological epidemic diseases. Controlling the malicious objects in computer network has been an increasingly complex issue in recent years. In order to curb the malicious object, we propose an $\mathrm{SI}_{1} \mathrm{I}_{2} \mathrm{RS}$ model for two different kinds of malicious groups. Hyman and Li $[1,2]$ proposed a DI (Differential- Infectivity) compartment for SIR model that describes the transmission dynamics of an infectious disease assuming infectious population divided into three compartments. Mishra et al. has introduced mathematical models for the transmission of malicious objects in the computer network and has given epidemic models on times delays, the fixed period of temporary immunity after the use of anti-malicious software, effect of quarantine, fuzziness of the system [3-7]. The action of malicious object throughout a network can be studied by using epidemiological models for disease propagation [8-13]. Richard and Mark [14] proposed an improved SEI (Susceptible - Exposed Infectious) model to simulate virus propagation. Anderson and May [15-16] discussed the spreading nature of biological viruses, parasite etc. leading to 
infectious diseases in human population through several epidemic models. Several authors have discussed stability of the system in epidemiology [17-25].

We develop a $\mathrm{SI}_{1} \mathrm{I}_{2} \mathrm{RS}$ model for different kind of infections that does not confer permanent immunity; infectious class has been divided into two sub compartments: first compartment for the worm infected nodes and second compartment for the virus infected nodes. Susceptible nodes go to the infectious class in accordance with the attacking behavior of malicious objects such as worm and virus and then move to the recovered class after the run of anti-malicious software. The model here have a variable total population size, because they have recruitment into the susceptible class by inclusion of some new nodes and nodes may crash due to the reason other than the attack of malicious objects and also due to the attack of malicious objects.

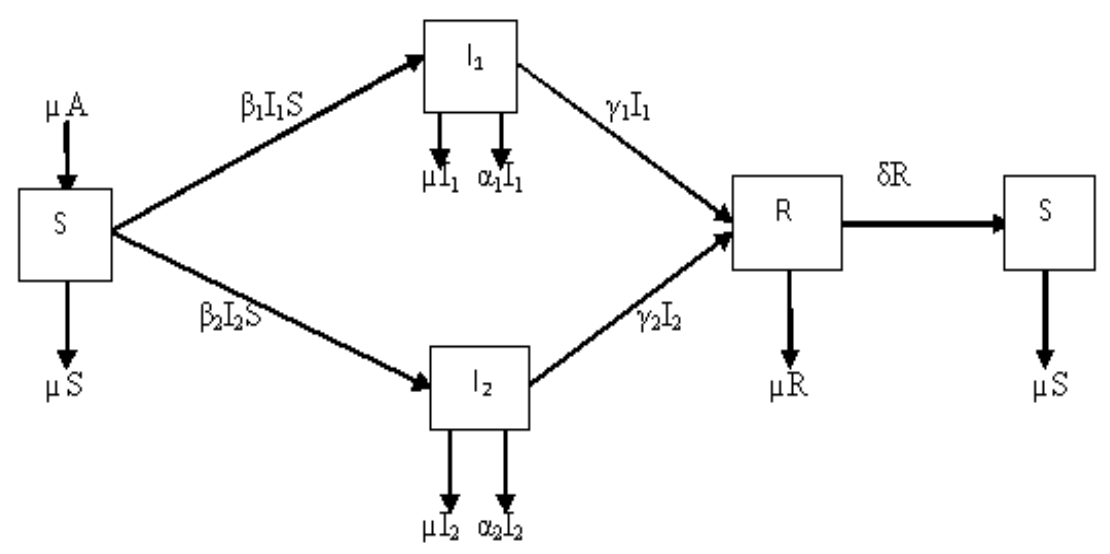

Figure 1. Schematic Diagram for the Flow of Malicious Objects in Computer Network

\section{2. $\mathrm{SI}_{1} \mathbf{I}_{2} \mathrm{RS}$ Model with Different Infectivity and Simple Mass Action Incidence}

Let $S(t)$ be the number of susceptible, $I_{1}(t)$ and $I_{2}(t)$ be the number of nodes infected by worm, and virus respectively, $\mathrm{R}(\mathrm{t})$ be the recover nodes after the run of anti malicious software and $N(t)$ be the total population size. Based on our assumption and flow of transmission as depicted in figure 1 , we have the following system of differential equations:

$$
\begin{gathered}
\frac{d S}{d t}=\mu(A-S)-q_{j} \sum_{j=1}^{2} \beta_{j} I_{j} S+\delta R \\
\frac{d I_{j}}{d t}=q_{j} \sum_{j=1}^{2} \beta_{j} I_{j} S-\left(\mu+\alpha_{j}+\gamma_{j}\right) I_{j}+v_{j} ; j=1,2 . \\
\frac{d R}{d t}=\sum_{j=1}^{2} \gamma_{j} I_{j}-(\mu+\delta) R
\end{gathered}
$$

where $q_{j}$ is the probability of infective nodes which enter into the group $I_{j}$ from the susceptible class. A is the recruitment of susceptible nodes in the computer network, $\mu$ is the per capita birth rate and death rate due to the reason other than the attack of malicious objects, $\gamma_{1}$ and $\gamma_{2}$ are the rates of nodes leaving the infectious class $I_{1}$, and $I_{2}$ to the recover class respectively. $\alpha_{1}$ and $\alpha_{2}$ are the crashing rate of the nodes due to the attack of malicious objects in infectious class $I_{1}$ and $I_{2}$ respectively. $\delta$ is the rate of transmission of nodes from recovered class to susceptible class.

Let, $\mathrm{N}=\mathrm{S}+\mathrm{I}_{1}+\mathrm{I}_{2}+\mathrm{R}$ 


$$
\frac{d N}{d t}=\mu A-\mu N-q_{j} \sum_{j=1}^{2} \alpha_{j} I_{j}
$$

\section{Malicious Objects Free Endemic Equilibrium Stage}

In the absence of malicious objects, when $t \rightarrow \infty, N \rightarrow A$.

Let us define the solution region in the domain $G$ given by $G=\left\{\left(S, I_{j} R\right) \in R^{4} / S \geq 0 ; I_{j} \geq 0 ; R \geq 0 ; S+I_{j}+R \leq, A \forall j=1\right.$ to 2$\}$

The system (1) has the infectious-free equilibrium stage at $\mathrm{E}_{0}=(\mathrm{A}, 0,0,0,0)$.

The basic reproduction number is thus $R_{0}=A \sum_{j=1}^{2} \frac{\beta_{j} q_{j}}{\mu+\alpha_{j}+\gamma_{j}}$

We have an endemic equilibrium $\mathrm{E}^{*}=\left(\mathrm{S}^{*}, \mathrm{I}^{*}{ }_{1}, \mathrm{I}_{2}{ }^{*}, \mathrm{R}^{*}\right)$, where,

$$
S^{*}=\frac{A}{R_{0}} ; I_{j}^{*}=\frac{\mu A\left(R_{0}-1\right) \gamma_{j}(\mu+\delta)}{\left\{\beta_{j}(\mu+\delta) A-\delta \gamma_{j} R_{0}\right\} \gamma_{j}} ; R^{*}=\frac{\mu A\left(R_{0}-1\right) \gamma_{j}}{\beta_{j}(\mu+\delta) A-\delta \gamma_{j} R_{0}}
$$

\section{Theorem 1}

Consider the system (1). If $R_{0}<1$ then solution set $G=\left\{\left(S, I_{1}, I_{2}, R\right) \in R^{3}: S \geq 0 ; \quad I_{j} \geq\right.$ $\left.0 ; S+I_{1}+I_{2}+R \leq A\right\}$ is locally asymptotically stable for infection free equilibrium $E_{0}$.

Proof: To show the local stability of the system (1), we construct the Jacobian matrix of (1) for the infection free equilibrium $\mathrm{E}_{0}$.

$$
J_{0}=\left[\begin{array}{ccc}
-\mu & D_{12} & D_{13} \\
0 & D_{22} & 0 \\
0 & D_{32} & -(\mu+\delta)
\end{array}\right]
$$

$$
\text { where } D_{22}=\left[\begin{array}{cc}
-\left(\mu+\alpha_{1}+\gamma_{1}\right)+q_{1} \beta_{1} S & q_{1} \beta_{1} S \\
q_{2} \beta_{1} & -\left(\mu+\alpha_{2}+\gamma_{2}\right)+q_{2} \beta_{2} S
\end{array}\right]
$$

Since

$$
\begin{aligned}
& R_{0}=A \sum_{j=1}^{2} \frac{\beta_{j} q_{j}}{\left(\alpha_{j}+\mu+\gamma_{j}\right)}<1 \\
& \Rightarrow\left(\mu+\alpha_{j}+\gamma_{j}\right)+q_{j} \beta_{j}<0 \forall j=1,2
\end{aligned}
$$

This implies that $\mathrm{D}_{22}$ is negative.

From the above Jacobian matrix $\mathrm{J}$, we see that the diagonal elements are real and negative. Therefore, the system is locally asymptotically stable.

\section{Theorem 2}

For the system (1), the infection free equilibrium $E_{0}$ is globally asymptotically stable in the solution set $G=\left\{\left(S, I_{1}, I_{2}, R\right) \in R^{4}: S \geq 0 ; I_{j} \geq 0 ; R \geq 0 ; S+I_{1}+R \leq A\right\}, \quad$ if $R_{0}<1$.

Proof: We define vectors $\mathrm{B}=\left(\beta_{1}, \beta_{2}\right)^{\mathrm{T}}, \mathrm{D}=\operatorname{diag}\left(\sigma_{1}, \sigma_{2}\right)$ and $\mathrm{Q}^{\mathrm{T}}=\left(\mathrm{q}_{1}, \mathrm{q}_{2}\right)$.

Where

$$
\begin{aligned}
& \sigma_{1}=\mu-\alpha_{1}+\gamma_{1} \\
& \sigma_{2}=\mu-\alpha_{2}+\gamma_{2}
\end{aligned}
$$

$\mathrm{I}=\left(\mathrm{I}_{1}, \mathrm{I}_{2}\right)^{\mathrm{T}}$ and $\mathrm{Y}=\left(\gamma_{1}, \gamma_{2}\right)$

System (1) can be written as: 


$$
\begin{aligned}
& \frac{d S}{d t}=\mu(A-S)-B^{T} I S+\delta R \\
& \frac{d I}{d t}=Q^{T} B^{T} I S-D I \\
& \frac{d R}{d t}=Y I-(\mu+\delta) R
\end{aligned}
$$

where $\mathrm{I}=\left(\mathrm{I}_{1}, \mathrm{I}_{2}\right)^{\mathrm{T}}$ and $\mathrm{Y}=\left(\gamma_{1}, \gamma_{2}\right)$. We express the basic reproduction number as $R_{0}=A B^{T} Q^{T} D^{-1}$.

Let us define the Liapunov function $V=B^{T} D^{-1} I$. Then $V$ is the positive definite for $I_{j} \geq$ 0 .

$$
\begin{aligned}
& \frac{d V}{d t}=\frac{d}{d t}\left(B^{T} D^{-1} I\right) \quad=\quad B^{T} D^{-1} \frac{d}{d t} I=B^{T} D^{-1}\left(Q^{T} B^{T} I S-D I+v\right)= \\
& \left(Q^{T} D^{-1} B^{T} S-1+D^{-1} v\right) B^{T} I \\
& =\left(\frac{R_{0}}{A} S-1\right) B^{T} I \leq 0 \text { where } \mathrm{S} \leq \mathrm{A} \text { and } \mathrm{R}_{0}<1 .
\end{aligned}
$$

Hence, the infection free equilibrium $\mathrm{E}_{0}$ is the only positive invariant in $\left\{\left(S, I_{j} R\right) \in G ; \frac{d V}{d t}=0 ;\right.$ for $\left.I=0\right\}$.

Therefore, it follows from the Liapunov stability theory that the infection free equilibrium $\mathrm{E}_{0}$ is globally asymptotically stable.

Table 1. Parametric Values used in Simulation

\begin{tabular}{|l|l|}
\hline Parameter & Value \\
\hline $\mathrm{N}$ & 17,000 \\
\hline $\mathrm{S}(0)$ & 9,500 \\
\hline $\mathrm{I}_{1}(0)$ & 1000 \\
\hline $\mathrm{I}_{2}(0)$ & 2,000 \\
\hline $\mathrm{R}(0)$ & 0 \\
\hline $\mathrm{A}$ & 0.009 \\
\hline$\mu$ & 0.05 \\
\hline$\beta_{1}$ & 0.001 \\
\hline$\beta_{2}$ & 0.005 \\
\hline$\delta$ & 0.005 \\
\hline $\mathrm{q}_{1}$ & 0.26 \\
\hline $\mathrm{q}_{2}$ & 0.27 \\
\hline$\alpha_{1}$ & 0.992 \\
\hline$\alpha_{1}$ & 0.889 \\
\hline$\gamma_{1}$ & 0.008 \\
\hline$\gamma_{2}$ & 0.007 \\
\hline
\end{tabular}




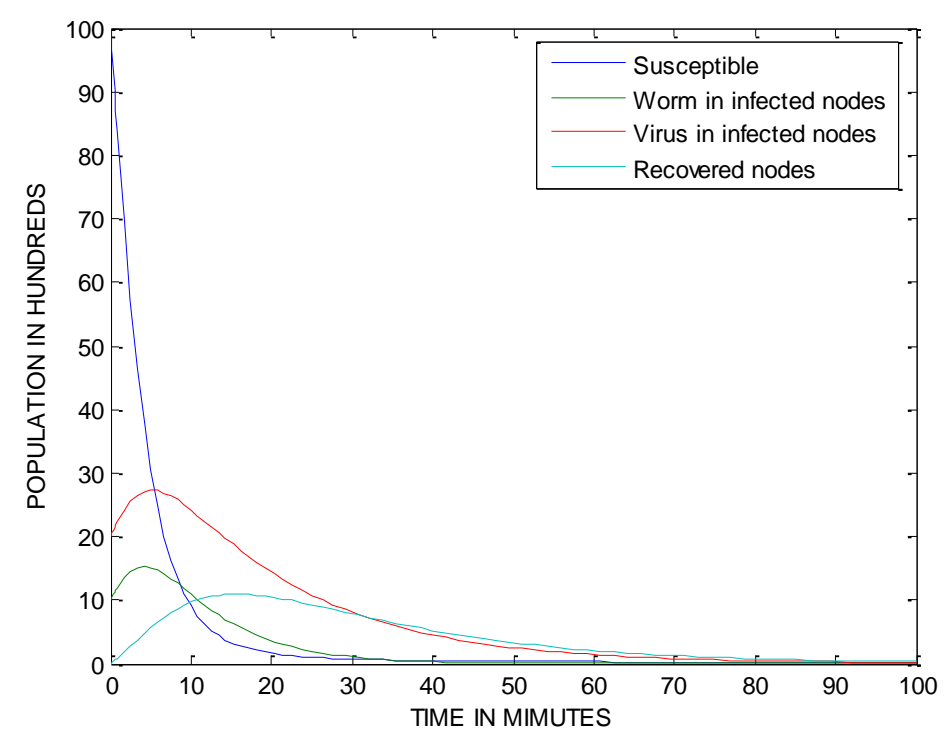

Figure 2. Dynamical Behavior of the System (1)

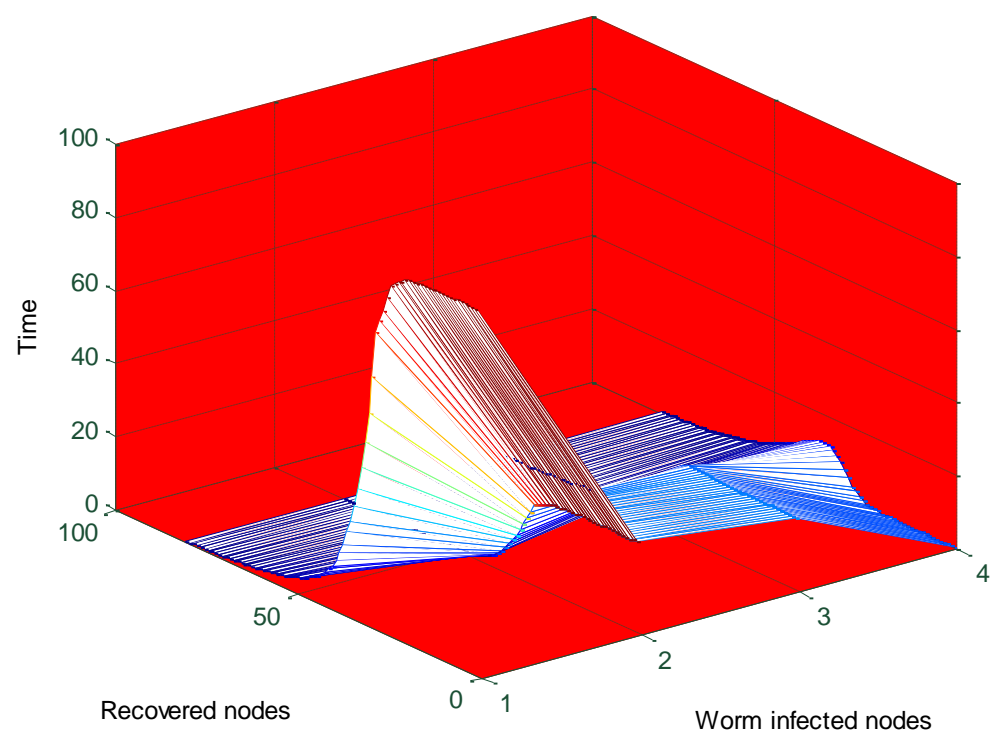

Figure 3. Dynamical Behavior of Worm Infected Nodes and Recovered Nodes with Time 


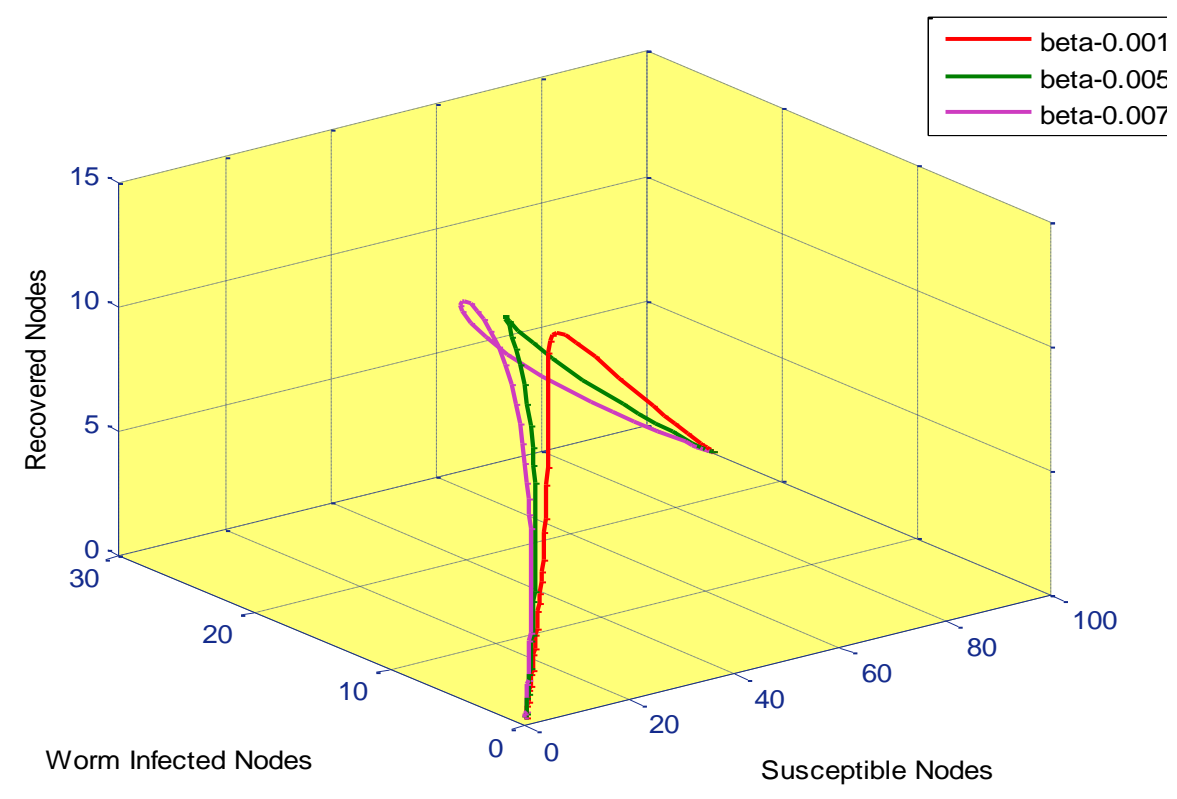

Figure 4. Transformation of Nodes from Recovered Class to Susceptible Class When Attacked By Worms

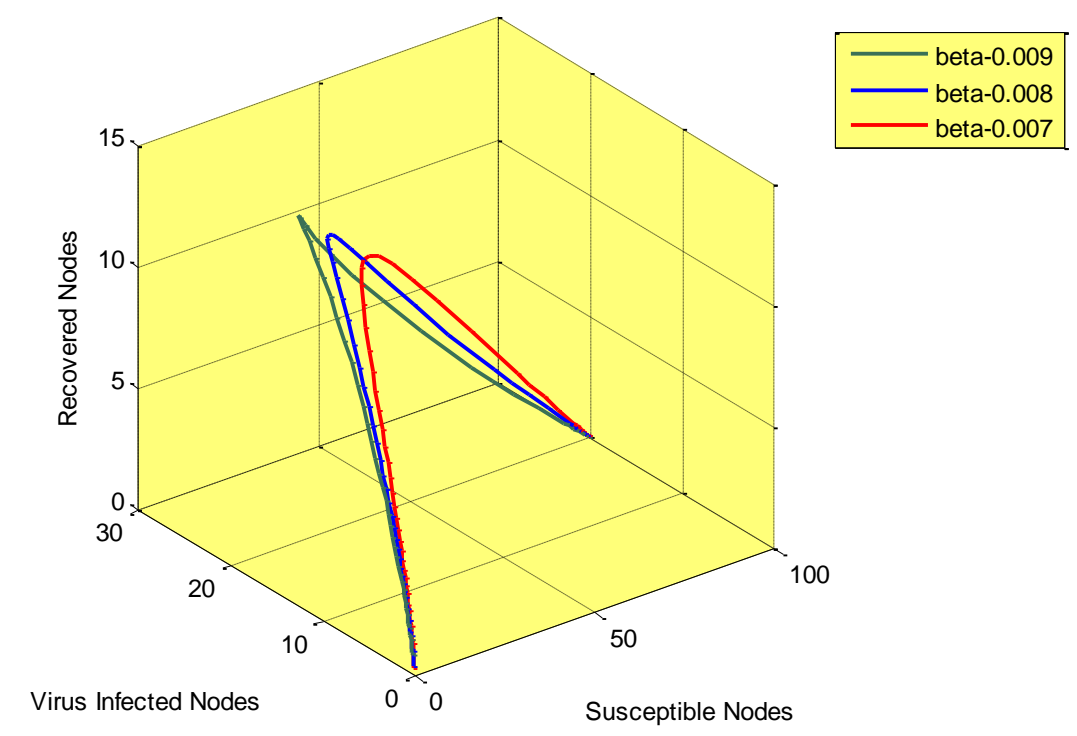

Figure 5. Transformation of Nodes from Recovered Class to Susceptible Class When Attacked By Viruses 


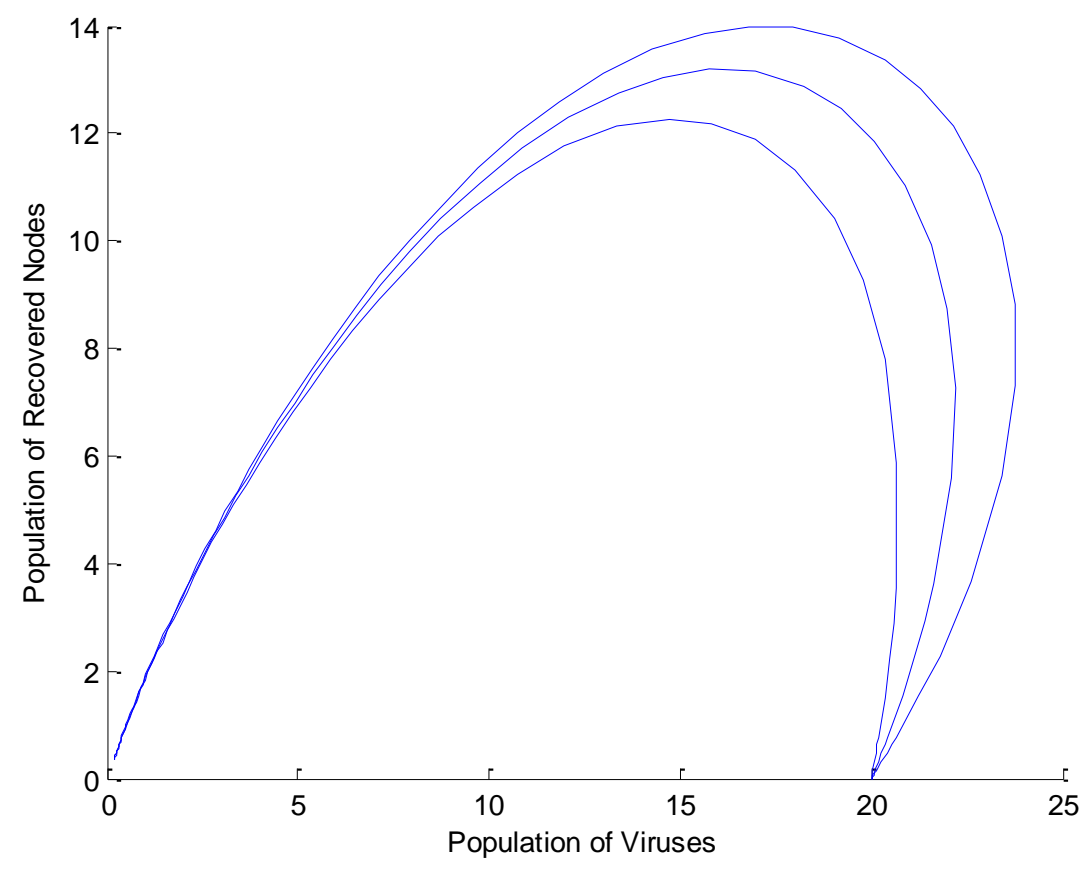

Figure 6. Effect of Virus Infected Nodes $I_{2}$ on Recovered Nodes $R$

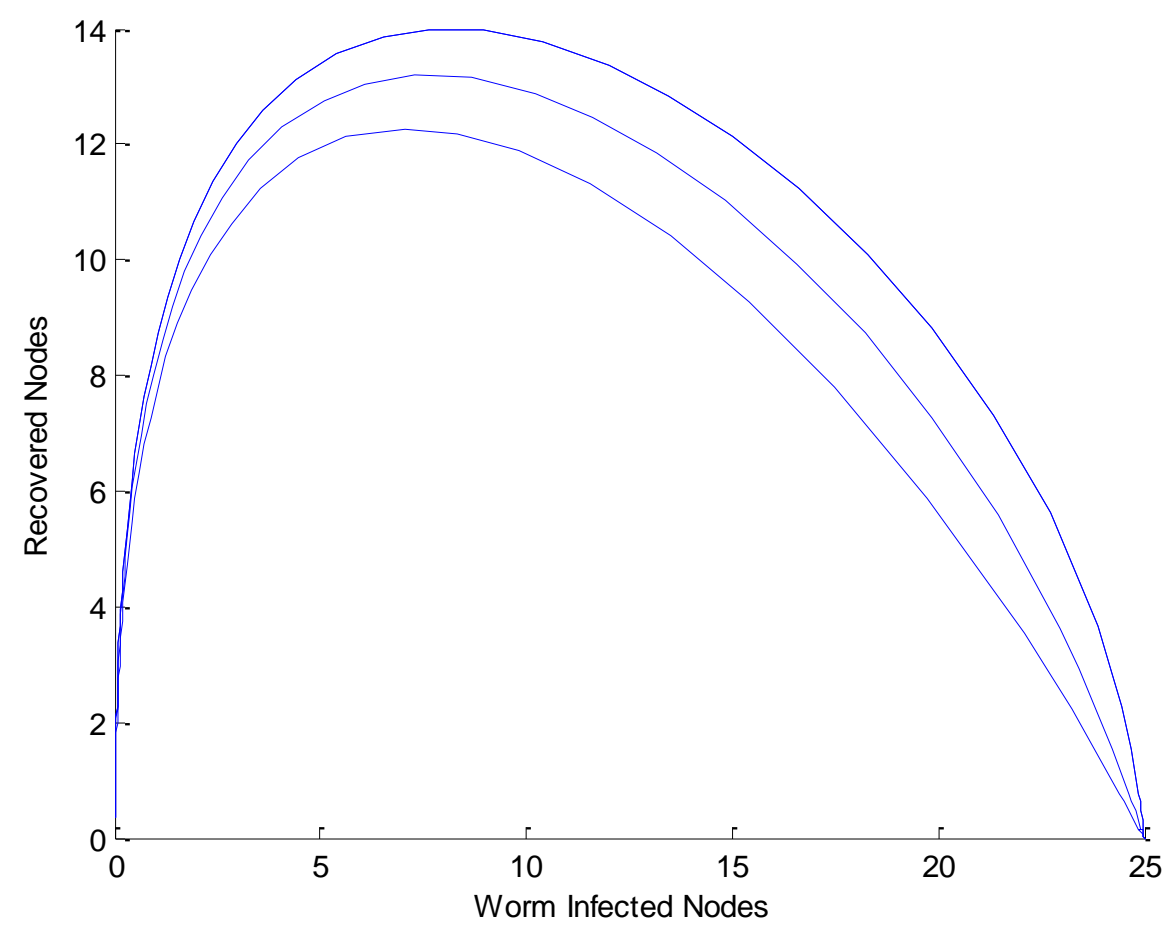

Figure 7. Effect of Worm Infected Nodes $I_{1}$ on Recovered Nodes $R$

\section{Conclusion}

A DI (differential infectivity) compartment for $\mathrm{SI}_{1} \mathrm{I}_{2} \mathrm{RS}$ epidemic model for the simple mass action incidence rate in which infected population is divided into two groups where first group consists of those nodes which are infected by the worms and second for the 
nodes which infected by virus is developed. We discuss the characteristics of the threshold parameter and established that if $\mathrm{R}_{0} \leq 1$, the system is locally asymptotically stable and globally asymptotically stable with help of Liapunov function. Runge-Kutta Fehlberg fourth fifth order method is used to solve and simulate the system by using real parametric values. Eventually, we find that with the decrease of susceptible and all kind of infection, recovered nodes increases due to the run of antivirus software and these are asymptotically stable with respect to time, which is depicted in Figure 2. Furthermore, analysis and simulation results show some managerial insights that are helpful for the practice of antivirus software in information sharing networks. The transformation of nodes from recovery class to susceptible class from worms and viruses is depicted in figures 3, 4, 5, 6 and 7 respectively which clearly indicates the immunity of the system due to the different attacks. The efficiency of the antivirus software can be analyzed by (i) observing the rate through which it cleans the nodes on attack of different kind of malicious objects, (ii) the rate through which the nodes crashes due to the attack of malicious objects. This will help the software organization to develop more efficient antivirus software that can minimize the attack and maximize the rate of recovery.

\section{References}

[1] J. M. Hyman, L. Jia and E. A. Stanley, "The Differentiated Infectivity and Staged Progression Models for the Transmission of HIV”, Math. Biosci, vol. 155, (1999), pp. 77-109.

[2] J. M. Hyman and L. Jia, "An Intuitive Formulation for the Reproductive Number for the Spread of Diseases in Heterogeneous Populations", Math. Biosci, vol. 167, (2000), pp. 65-86.

[3] B. K. Mishra and N. Jha, "SEIQRS Model for the Transmission of Malicious Object in Computer Network", Appl. Math. Model, vol. 34, (2010), pp. 710-715.

[4] B. K. Mishra and D. K. Saini, "SEIRS Epidemic Model with Delay for Transmission of Malicious Objects in Computer Network", Appl. Math. Comput. vol. 188, no. 2, (2007), pp. 1476-1482.

[5] B. K. Mishra and D. K. Saini, "Mathematical Models on Computer Virus", Appl. Math. Comput. vol. 187, no. 2, (2007), pp. 929-936.

[6] B. K. Mishra and N. Jha, "Fixed Temporary Immunity after run of Anti Malicious Software on Computer Nodes", Appl. Math. Comput. vol. 190, no. 2, (2007), pp. 1207-1212.

[7] B. K. Mishra and S. K. Pandey, "Fuzzy Epidemic Model for the Transmission of Worms in Computer Network", Nonlinear Analysis: Real World Applications, vol. 11, (2010), pp. 4335-4341.

[8] E. Gelenbe, "Dealing with Software Viruses: A Biological Paradigm", Inform. Secur. Tech. Rep, vol. 12, no. 4, (2007), pp. 242-250.

[9] E. Gelenbe, "Keeping Viruses under Control", in: Computer and Information Sciences_ISCIS 2005, 20th International Symposium, in: Lecturer Notes in Computer Science, Springer, vol. $373 \overline{3}$, (2005).

[10] E. Gelenbe, V. Kaptan and Yu Wang, "Biological Metaphors for Agent Behavior", in: Computer and Information Sciences_ISCIS 2004, 19th International Symposium, in: Lecturer Notes in Computer Science, Springer-Verlag, vol. 3280, (2004), pp. 667-675.

[11] J. R. C. Piqueira and F.B. Cesar, "Dynamical Model for Computer Virus Propagation, Mathematical Problems in Engineering", doi: 10.1155/2008/940526.

[12] J.R.C. Piqueira, B.F. Navarro and L.H.A. Monteiro, "Epidemiological Models applied to Virus in Computer Networks", J. Comput. Sc., vol. 1, no. 1, (2005), pp. 31-34.

[13] S. Forest, S. Hofmeyr, A.Somayaji and T. Longstaff, 'Self-nonself Discrimination in a Computer', in: proceedings of IEEE, Symposium on computer security and privacy, (1994), pp. 202-212.

[14] W. T. Richard and J. C. Mark, "Modeling Virus Propagation in peer-to-peer Networks", IEEE International Conference on Information, Communications and Signal Processing ICICS, (2005), pp. 981-985.

[15] R.M. Anderson and R. M. May, "Population Biology of Infection Disease”, Nature, vol. 180, (1999), pp. 361-367.

[16] R. M. Anderson and R. M. May, "Infection Disease of Humans; Dynamics and Control", Oxford University Press, Oxford, (1992).

[17] D. G. Halgh, "Hop Bifurcation in Epidemic Models with a Latent Period and Non Permanent Immunity", Math. Comput. Modell, vol. 25, (1997), pp. 85-107.

[18] H. W. Hethcote, H. W. Stech and P.van dan Driessche, "Periodicity and Stability in Endemic Models: a Survey", K. L. Cook(Ed.) Differential Equations and Applications in Ecology, Epidemics and Population Problems, Academic Press, New York, (1981), pp. 65-85.

[19] K. L. Cook and P.van den Drissche, "Analysis of SEIRS Epidemic Model with two Delays", Journal Math. Biol, vol. 35, (1996), pp. 240-260. 
[20] M. Y. L. J. R. Graff, L. C. Wang and J. Karsai, "Global Dynamics of a SEIR Model with a varying total Population Size”, Math. Biosci, vol. 160, (1999), pp. 191-213.

[21] M .Y. Li and J.S. Muldowney, "Global Stability for the SEIR Model in Epidemiology", Math. Biosci, vol. 125, (1995), pp. 155-164.

[22] M. Y. Li and L. Wang, "Global Stability in some SEIR Epidemic Models" C.C Chavez, S .Blower, P. Vanden Driessche, D. Kirscner and A. A Yakhdu (Eds), "Mathematical approaches for emerging and reemerging in infectious diseases", Models, Method and Theory, Springer, vol. 126, (2002), pp. 295312.

[23] Y. Michel, H. Smith and L. Wang, "Global Dynamics of SEIR Epidemic Model with Vertical Transmission", SIAM Journal Appl. Math, vol. 62, no. 1, (2001), pp. 58-69.

[24] J .K. Hale, “Ordinary Differential Equations", Second ed., Krieger, Basel, (1980).

[25] H. Hethcote, M. Zhein and L. Shengbing, "Effects of Quarantine in Six Epidemic Models for the Infectious Diseases", Math. Biosci, vol. 180, (2002), pp. 141-160.

\section{Author}

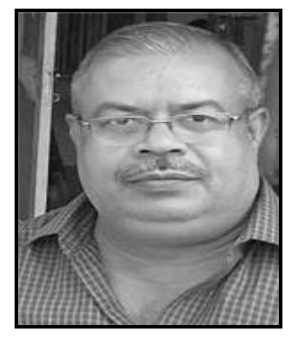

Binay Kumar Mishra, he is a Ph. D. in the early nineties from Ranchi University, Ranchi, India. He has published several research papers in journals of high repute and produced a dozen of Ph. Ds'. His research area includes fluid dynamics and nonlinear dynamics. 
International Journal of Future Generation Communication and Networking Vol. 9, No. 6 (2016) 